\title{
A Fluid Self-excited Oscillation Peculiar to Flat Ring Tube and its Application to Wearable Robots
}

\author{
Hideyuki Tsukagoshi, Ato Kitagawa, Keisuke Tambo, and Hiyoyuki Chiba, Tokyo Institute of Technology
}

\begin{abstract}
A novel fluid power actuator called Flat Ring Tube (FRT) is introduced in this paper. The mechanism of FRT is so simple that it only needs a urethane flat tube and some water pressure. No valves or switches are required. Applying constant water pressure results in periodic oscillation of the tube. The frequency is proportional to the flow rate of the water and inversely proportional to the tube length. By contacting the tube with a motor shaft, the motor rotates due to the periodic tube oscillation. Thus, we succeeded in rotating the motor without using any electricity. There are many possible applications with FRT, and we are developing a wearable massage device which makes people feel comfortable and improves their circulation of the blood.
\end{abstract}

\section{INTRODUCTION}

A fluid power actuator taking advantage of novel phenomenon will be proposed here. When the inside of a flat tube made of urethane is pressurized by domestic tap water pressure of approximately 0.2 to $0.3[\mathrm{MPa}]$, and the tube is bent in the round shape, then the self-excited oscillation occurs without using any valves to switch, as shown in Fig.1. This is the phenomenon peculiar to a flat tube bent in this form, which we call "Flat Ring Tube", abbreviated to "FRT" here. In this paper, a couple of its useful applications are shown after its basic driving principle is illustrated.

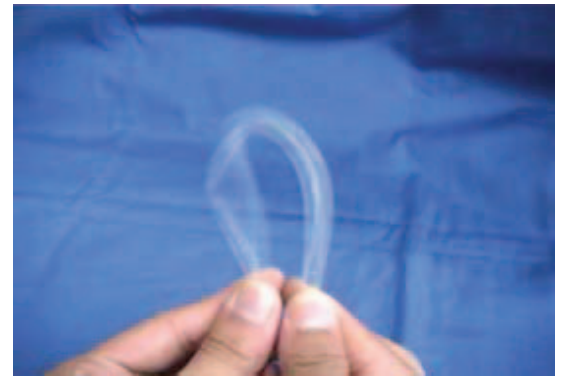

Fig.1 Overall view of the self-excited oscillation of FRT.

\section{BASIC PRINCIPLE}

The principle of the oscillation can be illustrated as follows. As the inside of the FRT is pressurized, it curves more and more and then it buckles at some point reaching the curvature

Manuscript received January 31, 2007.

Hideyuki Tsukagoshi is Associate Professor in Tokyo Institute of Technlogy (corresponding author to provide phone: +81-3-5734-3724; e-mail: htsuka@cm.ctrl.titech.ac.jp).

Ato Kitagawa is Professor in Tokyo Institute of Technlogy (corresponding author to provide phone: +81-3-5734-2550; e-mail: kitagawa@ cm.ctrl.titech.ac.jp). limit. Since the fluid passage is perfectly cut off by the buckled point, it is pushed from the upstream to the downstream as the tube continues to be pressurized, shown in Fig.2. At the same time, a new buckled point is also generated at the side of upstream, which follows the same trajectory as the previous point. This is a cycle of oscillation. The frequency of the oscillation by FRT is proportional to the flow rate, and it decreases as the tube becomes longer under the condition of the constant flow rate, shown in Fig.3.

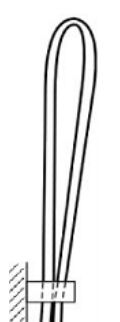

(1)

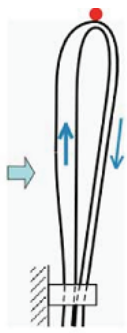

(2)

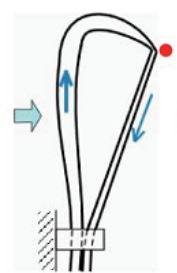

(3)

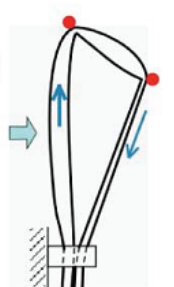

(4)

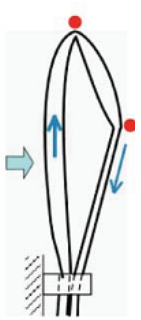

(5)
Fig.2 Principle of the periodic oscillation of FRT.

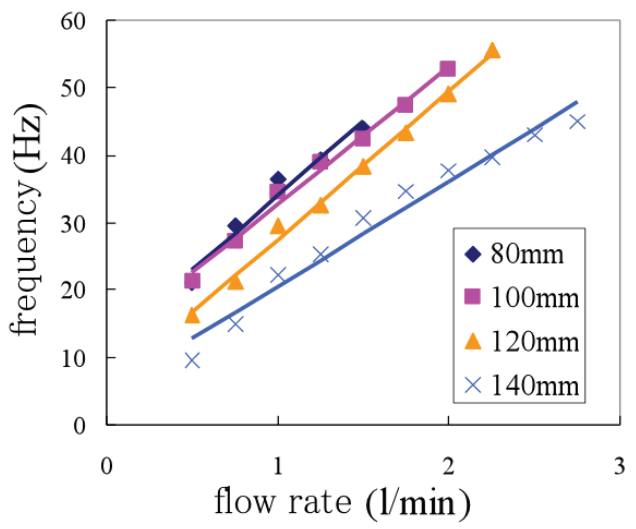

Fig.3 Experimental results suggesting the frequency related to tube length and the flow rate.

\section{APPLICATION TO A WATER HYDRAULIC MOTOR}

The oscillation by FRT is also able to generate the infinite rotary motion, shown in Fig.4. When a shaft supported by a bearing is touched by the inner side of FRT, it is rotated to one way, which is caused by the shifting contact point between the tube and the shaft. Since this rotary motion can be achieved even when FRT is bathed in water, this drive is useful as a water hydraulic motor by domestic tap water pressure applicable for the under water use. The developed motor, shown in Fig.5, could generate $10 \mathrm{~W}$ of output and 
$50 \mathrm{~g}$ in weight with extremely simple mechanism composed of a flat ring tube and a shaft.

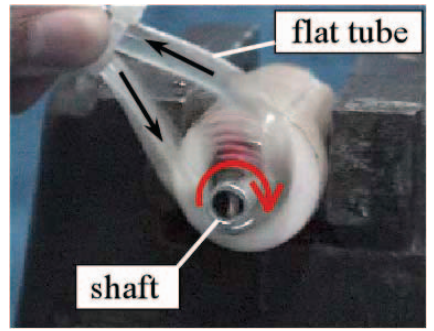

Fig.4 How to induce the infinite rotary motion.

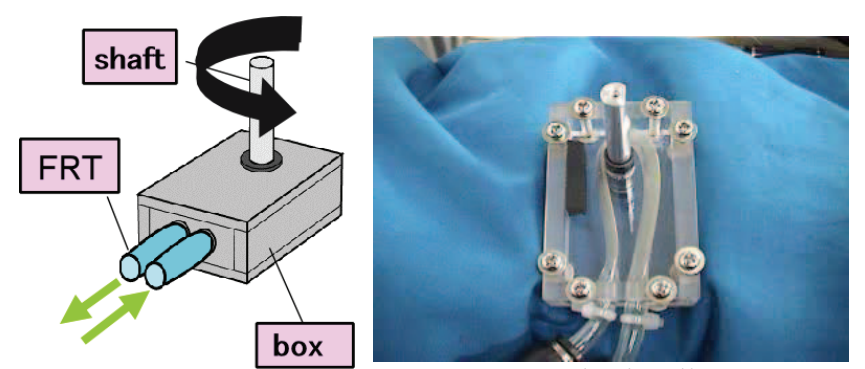

Fig.5 Application of FRT to a water hydraulic motor.

\section{Application to a LineAr Motion}

The free roller can be driven even when it is touched by the outer side of FRT. Therefore, if you mount it in the middle of a plate, you can make the plate kick the ground and go forward, as shown in Fig.6.
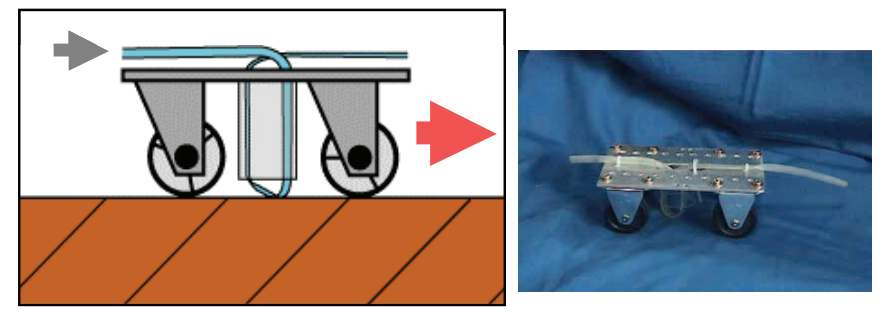

Fig.6 Application of FRT to a linear motion.

\section{Application to a Massage Device}

If you take advantage of this principle, it is expected to promote the blood flow. Then, we tried to apply it for massage devices, shown in Fig.7. Since it can generate the frequency of $0.5 \mathrm{~Hz} \sim 10 \mathrm{~Hz}$ with soft touchiness to human body, it can let them feel comfortable. In addition to the good feeling, the muscle blood flow meter showed that the muscle blood flow increased at $12 \%$ after 5 minutes massage, and that the effect of its increasing continues after 30 minutes, shown in Fig.8. The thermography also showed that the body temperature of the skin surface rose at $10 \%$ after 5 minutes massage by FRT. Unlike the conventional massage machine using the electricity, the FRT massage device does not generate the electromagnetic at all. Therefore, this device can be also applicable for a pacemaker user without any fear. At present, a wearable massage device is being developed which can massage shoulder, back and waist so as to promote the muscle pressure around the whole body.

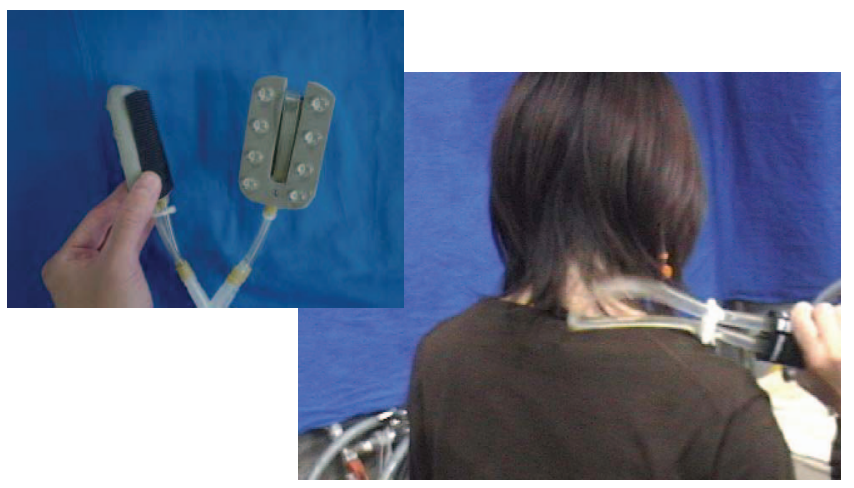

Fig.7 Two different types of the massage devices by FRT.

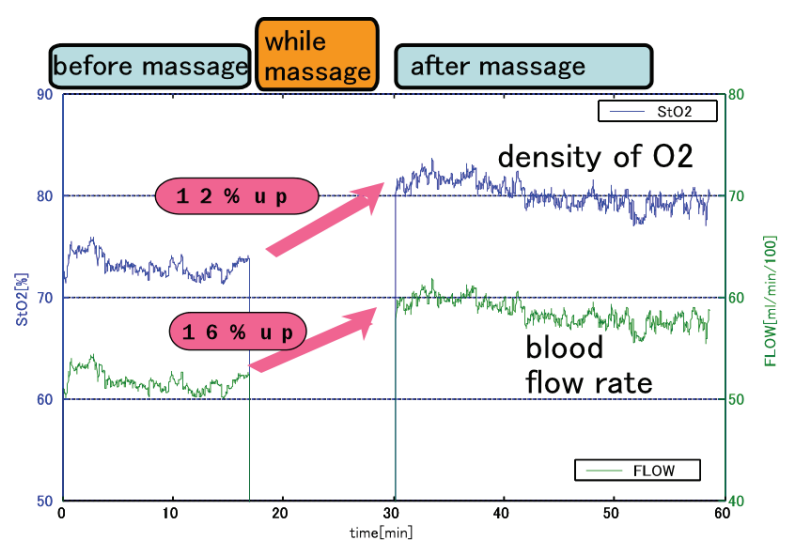

Fig.8 Effect of FRT to the human body.

\section{CONCLUSION}

This paper shows the principles and applications of a new fluid actuator based on a novel phenomenon. The practicality of the device is illustrated with experimental data showing the jacket mounted on the human body with the devices attached.

\section{ACKNOWLEDGMENT}

This study is financially supported by the ministry of education in the Japanese government. The project number related to this study is 17686020 of KAKEN-HI. We deeply thank to them.

\section{REFERENCES}

[1] H.Tsukagoshi, A.Kitagawa, Y.Kamata, "Wearable Fluid Power Composed of Transformed Flat Tube Actuators," IEEE/RSJ International Conference on Intelligent Robots and Systems, 1178-1183(2002)

[2] ] H.Tsukagoshi, S.Nozaki, A.Kitagawa : Versatile Water Hydraulic Motor Driven by Tap Water, IEEE / RSJ International Conference on Intelligent Robots and Systems,(CD-ROM:F-AII-2)(2000) 